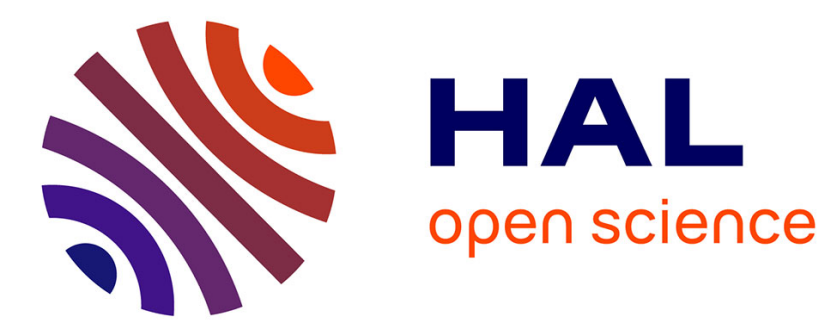

\title{
Thermodynamics of composition rules
}

T S Biró, K Urmössy, Z Schram

\section{To cite this version:}

T S Biró, K Urmössy, Z Schram. Thermodynamics of composition rules. Journal of Physics G: Nuclear and Particle Physics, 2010, 37 (9), pp.94027. 10.1088/0954-3899/37/9/094027 . hal-00600846

\section{HAL Id: hal-00600846 https://hal.science/hal-00600846}

Submitted on 16 Jun 2011

HAL is a multi-disciplinary open access archive for the deposit and dissemination of scientific research documents, whether they are published or not. The documents may come from teaching and research institutions in France or abroad, or from public or private research centers.
L'archive ouverte pluridisciplinaire HAL, est destinée au dépôt et à la diffusion de documents scientifiques de niveau recherche, publiés ou non, émanant des établissements d'enseignement et de recherche français ou étrangers, des laboratoires publics ou privés. 


\title{
Thermodynamics of Composition Rules
}

\author{
T S Biró ${ }^{1}, \mathrm{~K}$ Ürmössy ${ }^{1,2}$ and Z Schram ${ }^{3}$ \\ ${ }^{1}$ MTA KFKI Research Institute for Particle and Nuclear Physics, H-1525 Budapest \\ P.O.Box 49, Hungary \\ ${ }^{2}$ Eotvos Roland University, Budapest, Hungary, \\ ${ }^{3}$ University of Debrecen, H-4010 Debrecen P.O.Box 5, Hungary \\ E-mail: tsbiro@rmki.kfki.hu
}

\begin{abstract}
We review our recent approach to non-extensive thermodynamics based on general composition rules. We discuss arguments in favor of using such an approach for quark matter due to momentum-dependent interaction and present first results of attempts to compute the equation of state of su2 gluon matter on a lattice in this non-extensive, generalized canonical framework.
\end{abstract}

PACS numbers: 24.10.Pa, 25.75.Nq, 12.38.Gc

Submitted to: J. Phys. G: Nucl. Phys.

\section{Introduction}

Classical thermodynamics can be distilled into generally valid rules, into the so called four laws (from zeroth to third) of thermodynamics stating universal behavior of any system irrespective to the details of microscopic mechanisms - or the relativistic covariance properties of the underlying dynamics. Exactly this universality makes models based on this minimum of information so attractive for application to physical systems emerging after a rather complex dynamic evolution; among others to describe hadron spectra stemming from a fireball in high-energy heavy-ion collisions or, to an increasing degree, from more elementary hadronic reactions $[1,2,3,4,5]$.

As a new development of recent decades a strong wish has emerged to generalize classical thermodynamics to be valid for the non-extensive systems, where the classical requirement of having only short range correlations (and henceforth only short range interaction) in near-equilibrium systems is not fulfilled. Such systems are numerous in nature, like gravitating systems, charged plasmas, turbulent fluids, quantum entangled systems, nearly Bose-condensed atoms, and - important for our understanding of the very hadronization process in high-energy collisions - quark matter near to the critical point or near to the edge of mechanical stability. In non-extensive systems one or even all of the extensive variables, like entropy, internal energy, particle species number, volume, cannot be treated as such, because the correlation between large subsystems 
of thermodynamic bodies is not restricted to a thin layer near to their interface. To the contrary, corrections to the independent subsystem approximation are so important, that they do not vanish in the thermodynamic limit.

A novel approach to deal with such systems is still under vigorous investigation. Suggestions based on to use of an altered entropy formula instead of the BoltzmannGibbs-Shannon one trace back to the Hungarian mathematician Alfred Renyi, who has proved that there is a more general, extensive entropy formula than the classical one using the logarithm of probability $[6,7]$. An equivalent, but non-extensive entropy formula has been suggested in relation with informatics [8] and later in condensed matter physics by Constantino Tsallis [9, 10, 11, 12]. Discussions about the advantages and disadvantages of one or the other formula triggered ramified investigations of more formal nature: as a most important feature the Lesche-stability of the Renyi and Tsallis entropies was investigated $[13,14]$. Now it seems that these formulas can be used equivalently in statistical models and the physical meaning of the parameter $q$ in them is related to the degree of non-extensivity in the system under investigation.

Recently we have worked out a formal and simple approach to non-extensivity formulas based on the infinite repetition of composition rules generalizing the addition [15]. This procedure reveals that there is a way to treat statistical models with nonextensive properties mathematically clean. In the framework of the thermodynamics of abstract composition rules not only a general recipe is given for producing entropy formulas to each universal class of composition rules, but also suggestive generalizations of both the canonical equilibrium and the kinetic approaches are established $[16,17,18$, $19]$.

In this paper we review the basic formalism about the behaviour of repeated composition rules and the description of non-extensivity. We present then arguments about hadron $p_{T}$ spectra occurring in relativistic heavy-ion collisions showing nonextensive behaviour which does not seem to vanish with increasing participant number [20]. The use of this parameter expands the range of validity of statistical model fits to $p_{T}$ spectra dramatically. Also theoretical ideas will be discussed how relative momentum-dependent QCD interactions among partons may generate a non-additive rule for the individual kinetic energies causing this way a non-extensive statistical canonical distribution of hadron energies with a power-law tail. Finally, taking these hints seriously, we report about first attempts to obtain a non-extensive equation of state for $\mathrm{SU}(2)$ gluon matter in the framework of a numerical lattice gauge theory simulation.

\section{Composition rules and non-extensive formulas}

The classical Boltzmann-Gibbs-Shannon entropy formula,

$$
S=\sum f \ln \frac{1}{f}
$$

has the property that it is additive for independent probabilities

$$
S\left[f_{1} \cdot f_{2}\right]=S\left[f_{1}\right]+S\left[f_{2}\right] .
$$


The canonical energy distribution, $f=e^{-\beta E} / Z$, shows the back side of this coin: it is multiplicative by the addition of subsystem energies: $f\left(E_{1}+E_{2}\right)=f\left(E_{1}\right) \cdot f\left(E_{2}\right)$. In such systems the absolute temperature can be obtained as satisfying the relation $\beta=1 / T=\partial S / \partial E$ from the canonical requirement of maximizing the total entropy at fixed total energy: $S-\beta E=\max$. That the entropy is a useful concept is mainly reflected in the fact, that $\beta=1 / T$ occurs as an integrating factor rendering $d S$ to be a total differential (in contrast to the heat and mechanical work):

$$
d S=\frac{1}{T}(d E+p d V-\mu d N+\ldots) .
$$

Several physical mechanisms have since been suggested as micro-dynamical models to achieve and maintain a canonical equilibrium state [21]. The Langevin equation utilizes an additive white (Gaussian) noise for the momentum, the Fokker-Planck equation describes the linear diffusion process for the distribution of elementary momenta and Boltzmann's Stosszahlansatz incorporates the trend towards the irreversible growth of entropy.

All these mechanisms have already been generalized to deal with more entangled situations: the Langevin equation with multiplicative noise leads to a power-law tailed stationary distribution in kinetic energy [22], the anomalous (nonlinear) diffusion can establish the more general Levy or the t-Student distribution instead of the Gaussian, and the Stosszahlansatz also can be generalized exactly by manipulating the product assumption (or by manipulating the energy addition assumption valid for a separated pair of particles in a micro-collision) $[16,19]$. What is common in all these approaches can be understood as "deforming" the usual formulas using the logarithm and the exponential functions: in a way by keeping the same structure exactly these functions are replaced by their "deformed logarithm" and "deformed exponential" versions.

Starting with an abstract composition rule generalizing the simple addition,

$$
x \oplus y=h(x, y),
$$

its infinite repetitive use, $x_{n}=h\left(x_{n-1}, \Delta y_{n}\right)$, starting with zero $\left(x_{0}=0\right)$ and composing infinitesimally small quantities, - which nevertheless sum up to a finite value, $y=$ $\sum_{n} \Delta y_{n}$, - one can prove that the resulting composed quantity, $x=\lim _{n \rightarrow \infty} x_{n}$ satisfies the scaling differential equation

$$
\frac{d x}{d y}=\left.\frac{\partial}{\partial y} h(x, y)\right|_{y=0^{+}} .
$$

Its solution, $y=L(x)$ is the formal logarithm which maps the composition rule to the simple addition:

$$
x\left(y_{1}+y_{2}\right)=L^{-1}\left(L\left(x\left(y_{1}\right)\right)+L\left(x\left(y_{2}\right)\right)\right)
$$

is the asymptotic composition rule in the thermodynamic (infinite repetition) limit. Such asymptotic rules are associative and are attractors among all abstract composition rules (for details see $[15,19]$ ). 
A scaling parameter, $a$, can be used to interpolate between the simple addition and the asymptotic rule by introducing $L_{a}(x)=L(a x) / a$. Now $L_{0}(x)=x, L_{1}(x)=L(x)$ and the deformed exponential function is given by $e_{a}(x)=\exp \left(L_{a}(x)\right)$. Its inverse function is the deformed logarithm $\ln _{a}(x)=L_{a}^{-1}(\ln (x))$. Entropy composition formulas then satisfy the rule $L(\sigma(a b))=L(\sigma(a))+L(\sigma(b))$ and hence $\sigma(p)=\ln _{a}(1 / p)$. Considering now a generalized canonical problem a non-additive entropy is maximized while a non-additive total energy is kept fixed:

$$
\begin{aligned}
& Y\left(S_{1}\right)+Y\left(S_{2}\right)=Y\left(S_{12}\right)=\text { max. } \\
& X\left(E_{1}\right)+X\left(E_{2}\right)=X\left(E_{12}\right)=\text { const. }
\end{aligned}
$$

This construction also satisfies the zeroth law of thermodynamics because the requirement of canonical equilibrium factorizes to quantities containing data of one or the other system respectively [18]. This defines an absolute temperature as

$$
\beta=\frac{Y^{\prime}\left(S_{1}\right)}{X^{\prime}\left(E_{1}\right)} S_{1}^{\prime}\left(E_{1}\right)=\frac{Y^{\prime}\left(S_{2}\right)}{X^{\prime}\left(E_{2}\right)} S_{2}^{\prime}\left(E_{2}\right) .
$$

The relation between entropy, energy and temperature, however, is more complex than in the classical case. Examples for different composition rules and emerging entropy formulas can be constructed, for a list see the reference [19].

\section{Non-extensive hadron spectra}

A remarkable composition rule is defined by $h(x, y)=x+y+G(x y)$, where the asymptotic rule becomes $\varphi(x, y)=x+y+$ axy with $a=G^{\prime}(0)$. This is exactly the rule underlying the Tsallis and Renyi entropies. We have suggested in [15] that in the high kinetic energy kinematics, the individual kinetic energy composition rule may be deformed due to the momentum dependence of the interaction energy, $h\left(E_{1}, E_{2}\right)=E_{1}+E_{2}+U\left(Q^{2}\right)$ with $Q^{2}=2 E_{1} E_{2}(1-\cos \theta)$ containing the product of individual energies. This leads to a cut power-law asymptotic distribution in the non-extensive canonical state

$$
f_{\text {eq }}=\frac{1}{Z}\left(1+2 U^{\prime}(0) E\right)^{-\beta / 2 U^{\prime}(0)} .
$$

Unfortunately, $U^{\prime}(0)$ is an infrared property and is not readily available from perturbative QCD. By considering power-law tails in hadron spectra the phenomenological fits of this parameter is a possible starting point for further investigations.

In the framework of a quark coalescence picture we have analyzed RHIC mesonic and baryonic $p_{T}$ spectra and fitted them in a blast wave picture as possibly composed from a quark matter with $q \approx 1.2[23,24]$. Here we would like to demonstrate that the descriptive power of such a fit increases dramatically by including the non-extensivity parameter. In figure 1 the universality of an exponential fit for a collection of measured meson and baryon spectra (left side) is contrasted to that of a non-extensive fit based on the Tsallis-rule (right side). Not only the fit improves, but what is more important for 

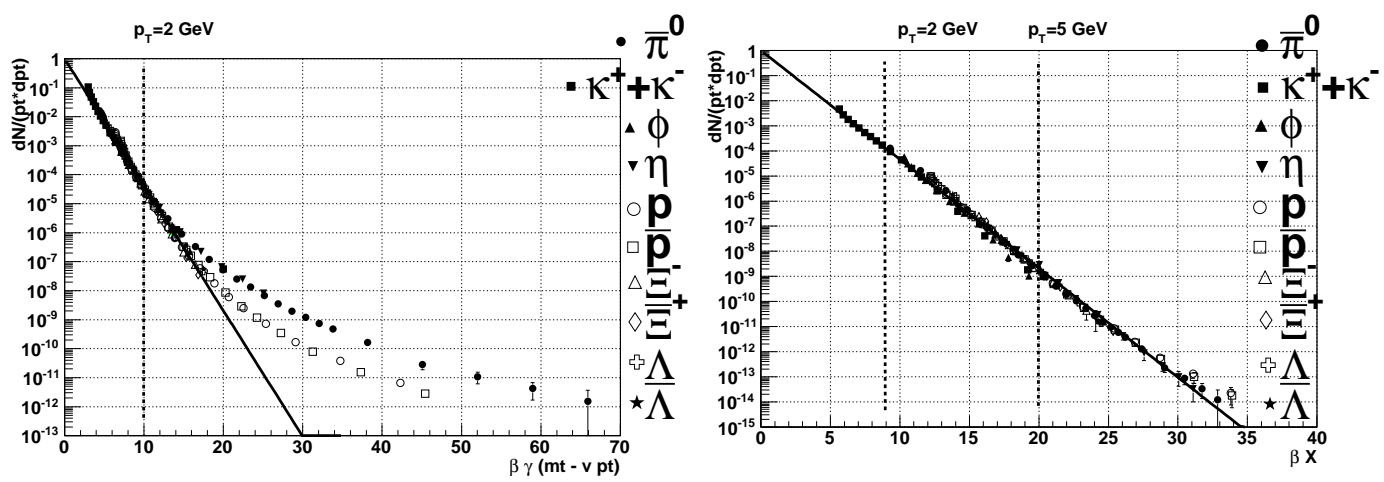

Figure 1. Scaling variable for RHIC $p_{T}$ distributions with transverse flow as a blast wave.

any thermal model, also its universality (dependence on hadron masses would directly deny the possibility of a state based on the stationarity energy distributions).

We note that applying power-law tailed distributions the energy density and pressure is higher at the same temperature than in the classical case. The energy density per degree of freedom for an ideal boson gas using the deformed exponential function with parameter $a$ is given by

$$
e=\frac{T^{4}}{2 \pi^{2}} \sum_{n=1}^{\infty} \frac{3 !}{n(n-a)(n-2 a)(n-3 a)} .
$$

Consequently aiming the same energy per particle values (which is determined by the experimental facts to be around $E / N \approx 1 \mathrm{GeV}$ in all relativistic heavy-ion experiments) the fitted values for the temperature for $q=1+a T>1$ are smaller than for $q=1$ ( $a=0$, the traditional case). For massless Boltzmann particles one easily obtains $3 T=E /(N+a E)<E / N$ for $a>0$.

\section{Non-extensive lattice $\mathrm{SU}(2)$ equation of state}

Motivated by the mathematical challenge and encouraged by the phenomenological success we raise the question: may it be that already the quark matter, preceding the hadronic fireball spectra, was in a non-extensive asymptotic state? The Monte Carlo method simulating lattice gauge field dynamics under non-extensive thermodynamic asymptotic conditions is based on the superstatistic interpretation [25, 26], where the power-law tailed Tsallis-Pareto distribution is convoluted from an Euler-Gamma distribution for the inverse temperature [17]. This in fact also has been interpreted as an effect of temperature fluctuations due to deficient equilibration in the fireball $[27,28]$. Since

$$
\frac{c^{c}}{\Gamma(c)} \int_{0}^{\infty} d t t^{c-1} e^{-c t} e^{-t \beta E}=\left(1+\frac{\beta E}{c}\right)^{-c},
$$

and the inverse temperature $\beta=1 / T=N a_{t}$ on the lattice is defined by its Euclidean time periodicity, we supplement the well-known lattice Metropolis method by sampling 


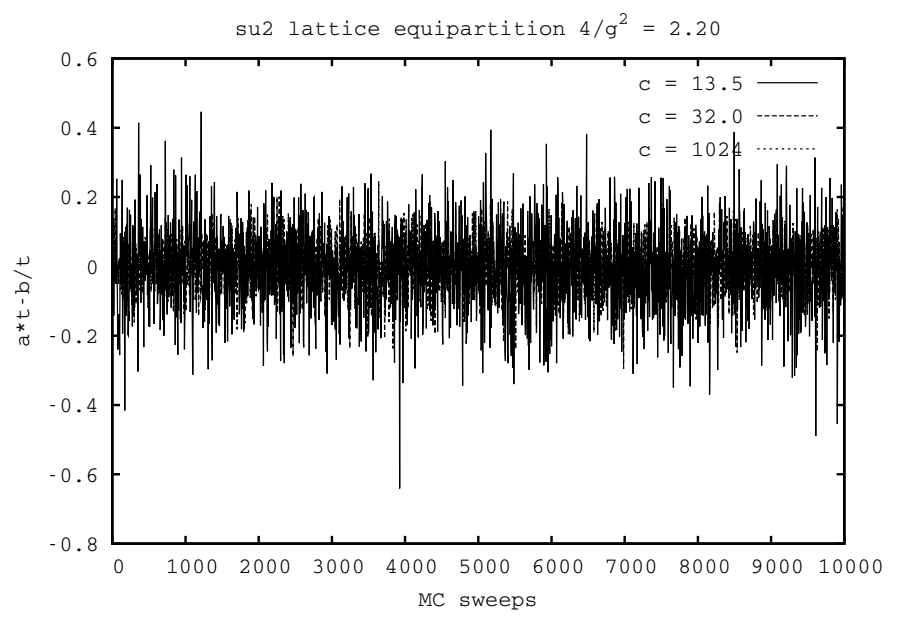

Figure 2. Equipartition of chromelectric and chromomagnetic contributions during the combined Euler-Gamma deviate and Metropolis method on a $10^{4}$ su2 lattice at $4 / g^{2}=2.10$.

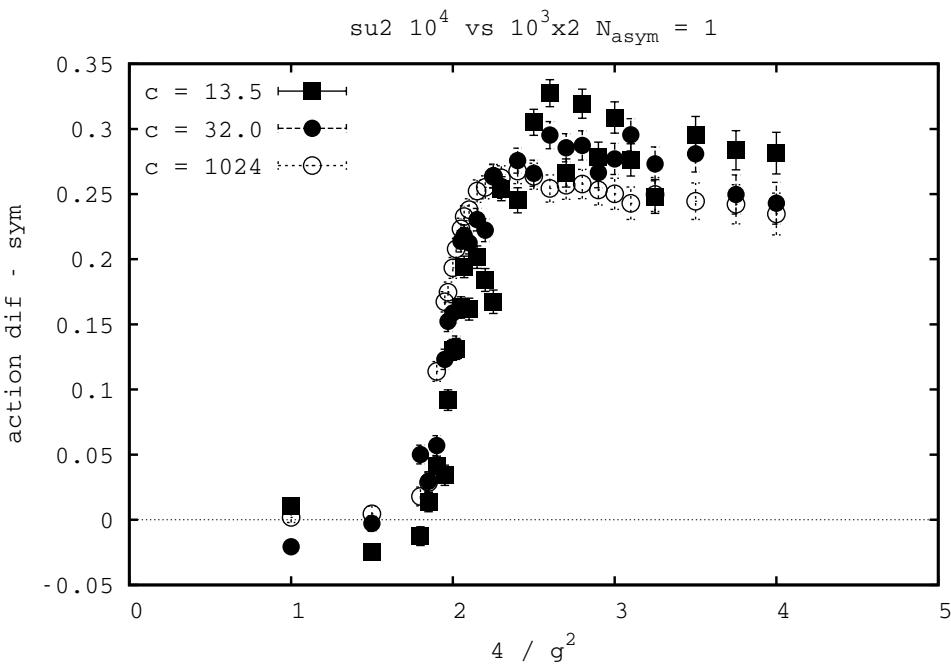

Figure 3. Action difference $(a-b)$ as a function of the inverse coupling $4 / g^{2}$ on an asymmetric $10^{3} \times 2$ lattice normalized to the symmetric lattice. These are the raw data for the scaled energy density, $e / T^{4}$ vs $T / T_{c}$ plot.

the asymmetry parameter (the ratio of timelike and spacelike lattice spacings), $t=a_{t} / a_{s}$, from an Euler Gamma distribution with the parameter $c$. This distribution stretches over non-negative values only and its width is $1 / \sqrt{c}$. We renew the parameter $t$ after each lattice sweep of throwing new link variables.

In figure 2 we present the equipartition between the chromoelectric and chromomagnetic plaquette average values corrected by the corresponding asymmetry factors: at equilibrates to $b / t$. A quick equilibration during the Monte Carlo process can be inspected for all different values of $c$ (1024, 32 and 13.5). 


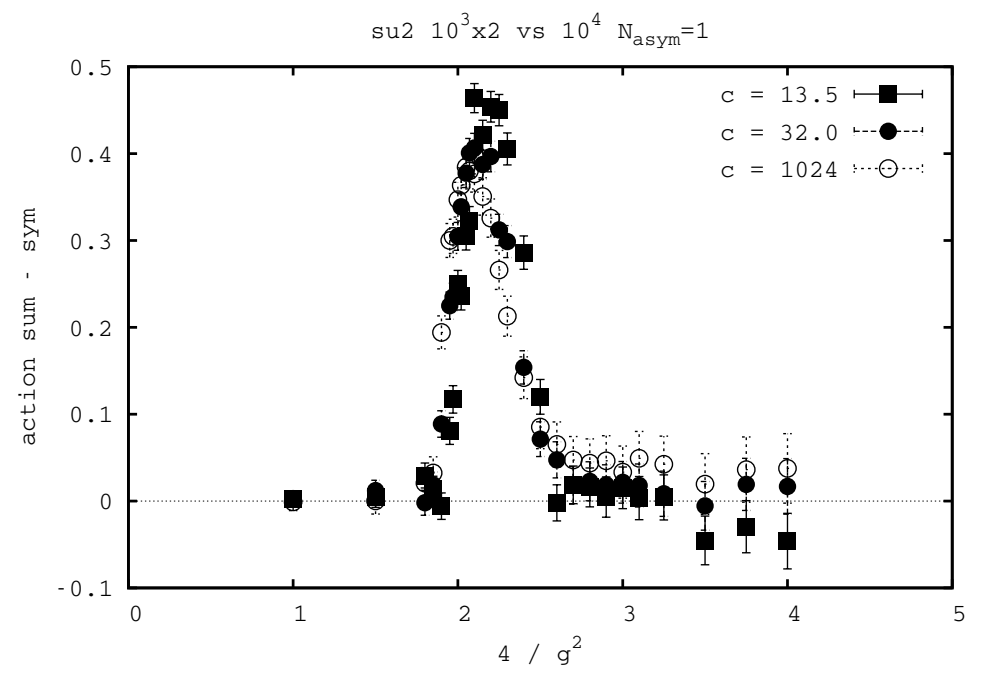

Figure 4. Action sum $(a+b)$ as a function of the inverse coupling $4 / g^{2}$ on an asymmetric $10^{3} \times 2$ lattice normalized to the symmetric lattice. These are the raw data for the interaction measure, $(e-3 p) / T^{4}$ vs $T / T_{c}$ plot.

Raw data towards obtaining the equation of state are collected from numerical simulations in figures 3 and 4 . The action difference between chromoelectric and chromomagnetic contributions on an asymmetric lattice is the basis of obtaining the scaled energy density $e / T^{4}$, while the action sum for the interaction measure $(e-3 p) / T^{4}$. Our results show already the main trends: smaller $c$ values - corresponding to bigger temperature fluctuations - increase the pressure and energy density. In fact, although numerical uncertainties are still large, a tendency can be observed for thickening the area under the action sum curve related to $(e-3 p) / T^{4}$. Of course further analysis has to be done and the renormalization of the physical temperature scale has to be carried out based on the static quark - antiquark potential analysis of Wilson Loop expectation values. The preliminary results presented here we regard as a first step into a promising direction.

In summarizing we have reviewed the thermodynamics of composition rules as a mathematical formulation for dealing with a non-extensive thermodynamic description, first of all formulas for entropy, energy and canonical distribution. We see the main reason for occurring power-law tailed distributions in certain parton cascade simulations and phenomenological fits to fireball hadron and quark matter spectra in the momentumdependent interaction term in QCD. This idea, of course, has to be solicited by further research. Finally we reported first results attempting to obtain a non-extensive equation of state for gluon matter in the framework of a modified Monte Carlo method in lattice simulation. Although first results look promising in this area, there is still a long way to go before the expected deviations from the classical thermodynamical picture can be pointed out by such numerical calculations. 


\section{Acknowledgments}

This work has been supported by the Hungarian National Science Fund OTKA (K49466, K68108 and K46925). Part of this work has been done within the framework of a joint research funded by the DFG and the Hungarian Academy of Sciences. Z.S. and T.S.B. are grateful to the Institue für Theoretische Physik, Johann Wolfgang Goethe Universität, Frankfurt for their hospitality.

\section{References}

[1] Hagedorn R 1965, Nuvo Cim. Suppl. 3147

[2] Cleymans J and Redlich K 1998, Phys. Rev. Lett. 815284

[3] Rafelski J and Letessier J 2000, Phys. Rev. Lett. 854695

[4] Torrieri G and Rafelski J 2004, J. Phys. G 30557

[5] Cleymans J, Hamar G, Levai P and Wheaton S 2008, Near-thermal equilibration with Tsallis distribution in heavy ion collisions, arXiv:0812.1471

[6] Renyi A 1959 Acta Math. Acad. Sci. Hung. 10193

[7] Renyi A, Probability Theory, North Holland, Amsterdam, 1970

[8] Daroczy Z, Aczel J, On measures of Information and their Characterization, Academic Press, New York, 1975

[9] Tsallis C 1988, J. Stat. Phys. 5250

[10] Tsallis C 1995, Physica A 221277

[11] Tsallis C 1999, Braz. J. Phys. 291

[12] Tsallis C, Eur. Phys. J. A 40257

[13] Abe S 2008, Eur. Phys. Lett. 84, 600006

[14] Van P and Matolcsi T 2009, On the continuity and Lesche stability of Tsallis and Renyi entropies and q-expectation values, arXiv:0910.1918

[15] Biro T S 2008, Eur. Phys. Lett. 8456003

[16] Biro T S and Purcsel G 2005, Phys. Rev. Lett. 95162302

[17] Biro T S, Purcsel G, Gyorgyi G, Jakovac A and Schram Z 2006, Nucl. Phys. A 774845

[18] Biro T S and Purcsel G 2008, Phys. Lett. A 3721174

[19] Biro T S, Purcsel G and Urmossy K 2009 Eur. Phys. J. A 40325

[20] Chinellato D D, Takahashi J and Bediaga I: A Non-extensive Equilibrium Analysis of Pion pt Spectra at RHIC, talk presented at SQM2009, Buzios, Rio, Brazil.

[21] Kodama T and Koide T 2009, Eur. Phys. J. A 40289

[22] Biro T S and Jakovac A 2005, Phys. Rev. Lett. 94132302

[23] Biro T S, Urmossy K, Barnafoldi G G 2008, J. Phys. G 35044012

[24] Biro T S, Urmossy K 2009, J. Phys. G 36064044

[25] Beck C, Cohen E G D 2003, Physica A 322267

[26] Beck C 2009, Eur. Phys. J. A 40267

[27] Wilk G, Wlodarczyk Z 2009, Eur. Phys. J. A 40299

[28] Wilk G, Wlodarczyk Z 2000, Phys. Rev. Lett. 842770 